\title{
Preliminary study on small angle X-ray scattering patterns of intact vancomycin susceptible and non-susceptible Staphylococcus aureus cells
}

\author{
Sujintana Wongthong ${ }^{\mathrm{a}, \mathrm{b}}$, Nuntaporn Kamonsutthipaijit ${ }^{\mathrm{c}}$, Cholthicha Kaewhan ${ }^{\mathrm{c}}$, \\ Aroonwadee Chanawong ${ }^{\mathrm{b}}$, Patcharaporn Tippayawat ${ }^{\mathrm{b}}$, Aroonlug Lulitanond ${ }^{\mathrm{b}, *}$ \\ a Faculty of Medical Technology, Nakhonratchasima College, Nakhon Ratchasima 30000 Thailand \\ b Centre for Research and Development of Medical Diagnostic Laboratories, Faculty of Associated Medical Sciences, \\ Khon Kaen University, Khon Kaen 40002 Thailand \\ c Beamline1.3W: Small Angle X-ray Scattering, Synchrotron Light Research Institute (Public Organization), \\ Nakhon Ratchasima 30000 Thailand
}

*Corresponding author, e-mail: arolul@kku.ac.th

Received 18 Feb 2021

Accepted 3 Aug 2021

\begin{abstract}
Low-level vancomycin-resistant $S$. aureus designated as heterogeneous vancomycin-intermediate $S$. aureus (hVISA) have been associated with treatment failure and unable to detect by routine disk diffusion method. As small angle X-ray scattering (SAXS) can be used for studying size, shape, and biology structure of bacterial cells; thus, this study aimed to investigate the SAXS patterns of biomolecules of vancomycin susceptible $S$. aureus (VSSA), hVISA, and, VISA cells. A total of 9 S. aureus isolates, 3 each from VSSA, hVISA, and VISA groups, were cultured on brain heart infusion agar with and without vancomycin. The cultured cells were kept overnight to reach their exponential phase and subjected to the SAXS using beamline 1.3W: SAXS. Under vancomycin untreated condition, the VISA cells showed different SAXS pattern from those of the VSSA and the hVISA, whereas the vancomycin treated cells of hVISA and VISA displayed similar SAXS patterns. The ribosome of VSSA was significantly smaller than that of hVISA under the untreated condition but showed no statistically different under the treated condition. In addition, when compared the ribosome and the DNA of VSSA with the VISA's and the DNA of VISA with the hVISA's, they were different only under the untreated condition. This preliminary study showed that under the stress condition mediated by vancomycin, the vancomycin non-susceptible $S$. aureus (hVISA and VISA) had similar SAXS patterns; while under the non-stress condition, the VSSA and hVISA showed similar patterns. This study provides preliminary information on bacterial adaptation under vancomycin-mediated stress condition.
\end{abstract}

KEYWORDS: Staphylococcus aureus, small angle X-ray scattering, biological macromolecules, vancomycin

\section{INTRODUCTION}

Staphylococcus aureus is the most common Grampositive cocci pathogen in community and health care associated infections especially the methicillinresistant $S$. aureus (MRSA) strains. It causes various infections including skin and soft tissue infections, pneumonia, bone infection, and blood stream infection [1]. Vancomycin is a main drug for treatment of serious MRSA infections. Wide uses of this antibiotic led to increasing vancomycin non-susceptible MRSA, resulting in the difficulty in clinical management [2, 3]. The low-level vancomycin-resistant $S$. aureus, such as vancomycin-intermediate $S$. aureus (VISA) and heterogeneous vancomycin-intermediate $S$. aureus (hVISA), had increased cell wall thickness which did not relate to any specific genetic determinant. They were typically associated with extended hospitalization and persisting bacterial infection, leading to prolong therapy and treatment failure [4-6].

Small angle X-ray scattering (SAXS) is an X-ray spectroscopy technique used for studying the size, shape, and biology structure on scale of 1 to 100 nanometers [7]. The application of SAXS has devel- oped during the past decade. The most application is the study of shape and function of macromolecules and nanocomposites in solutions [8]. For example, SAXS has been applied to examine human bone tissue, breast cancer tissue, micelles, and hemoglobin as well as to study the changing of intracellular structure of Escherichia coli cells induced by certain antibiotics $[9,10]$. Currently, there is no SAXS information of $S$. aureus especially among isolates with reduced susceptibility to vancomycin. Therefore, the application of SAXS in this study may provide scattering patterns of the morphological impact of vancomycin on $S$. aureus. Here, the X-rays scattering patterns of bacterial cell suspensions obtained from $3 \mathrm{~S}$. aureus groups: VSSA, hVISA, and VISA, were measured and, then, used to investigate bacterial intracellular components under the effect of vancomycin.

\section{MATERIALS AND METHODS}

Bacterial strains and culture conditions

Nine $S$. aureus isolates, 6 isolates derived from Srinagarind Hospital and 3 references strains, were used in this study (Table 1). The 9 isolates, all from frozen 
Table 1 Bacterial isolates and their half vancomycin MIC used in this study.

\begin{tabular}{llc}
\hline S. aureus strain & Phenotype & Half MIC $(\mu \mathrm{g} / \mathrm{ml})$ \\
\hline ATCC29213 & VSSA & 0.25 \\
MR2 & VSSA & 0.5 \\
MR3 & VSSA & 0.5 \\
ATCC700698 (Mu3) & hVISA & 1 \\
MR9 & hVISA & 0.5 \\
$70-97$ & hVISA & 0.5 \\
ATCC700699 (Mu50) & VISA & 2 \\
150 & VISA & 2 \\
127 & VISA & 1.5 \\
\hline
\end{tabular}

stocks, consisted of 3 isolates each of VSSA, hVISA, and VISA. They were first sub-cultured on blood agar (Oxoid, Hampshire, England), incubated at $37^{\circ} \mathrm{C}$ for $24 \mathrm{~h}$, and then grown on Tryptic Soy Agar (TSA: Oxoid, Thermo Fisher Scientific, MA, USA) with vancomycin concentration equal to half MIC of each isolate and without vancomycin. The TSA plates were incubated at $37^{\circ} \mathrm{C}$ for $24 \mathrm{~h}$. Each bacterial isolate was cultured on 3 sets of TSA plates representing 3 biological replicates.

The three reference strains of $S$. aureus: ATCC29213, ATCC700698 (Mu3), and ATCC700699 (Mu50), were controls for VSSA, hVISA, and VISA phenotypes, respectively.

\section{Minimum inhibitory concentration (MIC)}

The vancomycin MIC values of all isolates were determined using agar dilution method according to the CLSI 2019 [11].

\section{Modified population analysis profile with an area under the curve (PAP-AUC) for identification of hVISA phenotype}

A modified PAP-AUC was conducted according to Wootton et al [12]. Briefly, a serial ten-fold dilution of $0.5 \mathrm{McFarland}$ suspensions to $10^{-6}$ of each isolate was prepared. An aliquot of $20 \mu \mathrm{l}$ from each dilution was spread on brain heart infusion (BHI) (Oxoid) agar plate containing vancomycin (Sigma-Aldrich, MO, USA) concentrations of $0,0.5,1,2,3,4,5,6,7$, and $8 \mu \mathrm{g} / \mathrm{ml}$. After $48 \mathrm{~h}$ of incubation, the number of bacteria in each plate was mathematically calculated, and the results were presented as colony forming unit per milliliter (CFU/ml). Then, the $\log _{10}$ of $\mathrm{CFU} / \mathrm{ml}$ grown on each plate was plotted versus the vancomycin concentrations using the Graph Pad Prism software version 5.0.1 (GraphPad Software Inc., CA, USA). The PAPAUC ratio was calculated from areas under the curve of the test isolate and the reference hVISA strain (Mu3). The isolates having PAP-AUC ratios less than 0.9, 0.91.3 , and more than 1.3 were considered being VSSA, hVISA, and VISA phenotypes, respectively [12].

\section{Sample preparation for SAXS}

The bacterial cells from exponential phase culture were suspended in $0.1 \mathrm{M}$ phosphate buffer saline (PBS) $\mathrm{pH} 7.0$ at $10^{8} \mathrm{CFU} / \mathrm{ml}$, washed 3 times with Piperazine-N, $\mathrm{N}^{\prime}$-bis (2-ethanesulfonic acid) (PIPES) buffer (0.1 M, pH 7.0), and fixed with $2.5 \%$ glutaraldehyde solution in PIPES buffer for $1 \mathrm{~h}$. After centrifugation at $9000 \mathrm{rpm}$, the pellet was washed 3 times with PBS buffer ( $10 \mathrm{mM}$, pH 7.0), then resuspended in $100 \mu \mathrm{l}$ of PBS buffer and stored at $4^{\circ} \mathrm{C}$ until subjected to the SAXS experiment.

\section{Small angle X-ray scattering experiments}

The SAXS experiments were carried out at the beamline 1.3W: SAXS/WAXS of Synchrotron Light Research Institute (SLRI), Nakhon Ratchasima, Thailand. Beamline $1.3 \mathrm{~W}$ delivers a total photon flux of $2 \times 10^{9} \mathrm{Ph} / \mathrm{s}$ focused to a sample spot size of $2 \mathrm{~mm} \times 1 \mathrm{~mm}$ (horizontal $\times$ vertical) [13]. To get a homogeneous suspension, the bacterial cells were gently resuspended prior to the SAXS measurement. A $60 \mu \mathrm{l}$ sample of bacterial cell suspension was applied into a liquid cell, and the SAXS data were recorded using the Mar SX165 CCD detector at sample-detector distance of $4.6 \mathrm{~m}$ and at a wavelength of $1.38 \AA$. The range of momentum transfer $0.055<\mathrm{q}<1.28 \mathrm{~nm}^{-1}$ was covered $(\mathrm{q}=$ $(4 \pi \sin \theta) / \lambda$; where $2 \theta$ is the scattering angle, and $\lambda$ is the X-ray wavelength). Each sample solution was measured at $20^{\circ} \mathrm{C}$ and 10 min exposure time. Before measuring the sample, PBS buffer was measured as a background for each sample. The 2D SAXS images were reduced and radially averaged by the program SAXSIT, which was developed by SLRI staff, to obtain 1D scattering curves.

\section{Data analysis}

The 1D SAXS scattering profile, ploted between the intensity and the scattering vector q, was fitted to log normal size distribution to investigate size and size distribution of particle using software package SASfit produced by Paul Scherrer Institute (PSI) [14]. The form factor was used as a model of spherical particle and 3 sphere models were applied to fit the scattering profile. Three sphere models with decreasing sizes (from big to small) were used to represent ribosome, DNA, and small protein, respectively [10].

The paired $t$-test was used for statistical analysis to evaluate any difference between the size distribution of internal composition of $S$. aureus isolates grown in media with and without vancomycin (vancomycin treated and untreated conditions) (VSSA-(U) vs. VSSA(T), hVISA-(U) vs. hVISA-(T), and VISA-(U) vs. VISA(T)), while independent $t$-test was used to compare the size distribution of $S$. aureus between each phenotype (VSSA vs. hVISA, VSSA vs. VISA, and hVISA vs. VISA). The $p$-value of less than 0.05 was considered as statistically significant. 


\section{RESULTS}

\section{MIC and PAP-AUC of $S$. aureus}

The vancomycin MIC levels before and after culture in medium plus vancomycin of each isolate were equal. The MIC levels of individual 3 isolates of VSSA, hVISA, and VISA groups were $0.5-1,1-2$, and $3-4 \mu \mathrm{g} / \mathrm{ml}$, respectively; while their PAP-AUC were 0.51, 0.63, 0.67; $0.92,0.96,1.14$; and $1.46,1.58,1.62$, respectively (Table 1).

\section{Comparison of scattering patterns}

Among the cells of VSSA, hVISA, and VISA groups, the vancomycin-untreated cells of VSSA and hVISA showed similar SAXS patterns, but they were different from that of the VISA (Fig. 1A). In contrast, the vancomycin-treated cells of hVISA displayed SAXS patterns similar to that of the VISA-treated cells (Fig. 1B).

When comparing the scattering patterns between vancomycin treated and untreated cells within the VSSA, hVISA, and VISA groups; the patterns of VSSA, hVISA, and VISA cells after treatment with vancomycin were similar to that of their corresponding untreated cells (Fig. 2).

\section{Size distribution among VSSA, hVISA, VISA cells}

In the present study, we found that all bacterial isolates, including the reference strains, showed similar scattering patterns. These patterns yielded similar form factor which were fitted to a previous report [10]. The intracellular components of $S$. aureus isolates were modeled as filled spheres from literature data on the elementary composition and density [10]. In our results, a minimum of 3 populations were found: the largest size the ribosomes, followed by the DNA, and the small proteins. Each population had a mean diameter range of $58.80-92.20 \mathrm{~nm}, 11.00-17.10 \mathrm{~nm}$, and 1.40 $4.30 \mathrm{~nm}$, respectively (Table 2 ).

Comparing within each corresponding bacterial group, there was no difference in the size of each component between the vancomycin treated and untreated cells $(p>0.05)$. The treated VSSA and hVISA had mean ribosome size larger than that of the untreated cells, whereas the treated VISA had mean ribosome size smaller than the treated cells. However, none were statistically different $(p>0.05)$.

Comparing between the bacterial groups under vancomycin untreated condition, the ribosome of VSSA vs. hVISA, the ribosome and DNA of VSSA vs. VISA, and the DNA of VISA vs. hVISA were statistically different $(p<0.05)$. The ribosome of VSSA was smaller than those of hVISA and VISA. The DNAs of VSSA and hVISA were larger than that of VISA. In addition, the small proteins of VSSA were larger than those of hVISA and VISA. On the other hand, under the vancomycin treated condition, only small proteins of VSSA were significantly larger than those of VISA $(p<0.05)$ (Table 3$)$.

\section{DISCUSSION}

SAXS technique has been used to study various interesting model of bacteria. SAXS experiments using a synchrotron source are mostly optimized to study components with size ranges of around 1-100 nm [15]. A report of SAXS scattering of $S$. aureus cells showed a complex model assumed that phosphoglucosamine mutase enzyme (GlmM) inhibited di-adenylate cyclase enzymes (DacA) by masking the active site of the cyclase and preventing higher oligomer formation. This report provided an important mechanism of how cyclic di-adenosine monophosphate (c-di-AMP) production can be regulated in bacterial cell [16]. Study on an important virulence factor of $S$. aureus, Staphylococcal protein A (SpA) using SAXS provided the statistical conformation of a flexible protein [17]. It was found that using SAXS scattering to study ultrastructural changes in MRSA responding to broad-spectrum antimicrobial peptides was superior over electron microscope for contributing towards the development of drugs against resistant bacteria [18].

The SAXS scattering patterns of VSSA, hVISA, and VISA isolates between the vancomycin treated and untreated cells were not significantly different. This may be due to the low concentration of vancomycin (half MIC, Table 1) used that might not have any significant effect to these isolates. In contrast, there were significant differences on scattering patterns among the bacterial groups. Under the vancomycin untreated condition, VSSA and hVISA had similar scattering patterns which were different from that of VISA (Fig. 1A). This was in accordance with the phenotypic characteristics of VSSA and hVISA which expressed as vancomycin susceptible results (MIC 0.5$2 \mu \mathrm{g} / \mathrm{ml}$ ) when tested using the routine susceptibility method. However, the hVISA isolates, which had PAP-AUC value higher than that of the VSSA, were suggested to be a primary stage in the transformation from VSSA to VISA phenotypes [19]. Therefore, under the condition without vancomycin pressure, the hVISA isolates tended to have similar performance to that of the VSSA. On the other hand, under vancomycin treated condition, the hVISA cells had scattering pattern similar to that of the VISA (Fig. 1B) suggesting that the hVISA tended to have similar behavior to the VISA under vancomycin pressure. This may be due to their similarity in tolerance to low level vancomycin (both the hVISA and the VISA had higher PAP-AUC values than those of the VSSA) [12]. In addition, our previous study and a recent report showed that the hVISA isolates also had thickening cell wall, though not as thick as that of the VISA isolates $[20,21]$. The vancomycin MIC of the VISA isolates in this study was slightly higher than those of the hVISA and the VSSA isolates. It has been reported that the cell wall thickness of $S$. aureus had high correlation with the vancomycin MIC [22]. Thus, the vancomycin MIC 

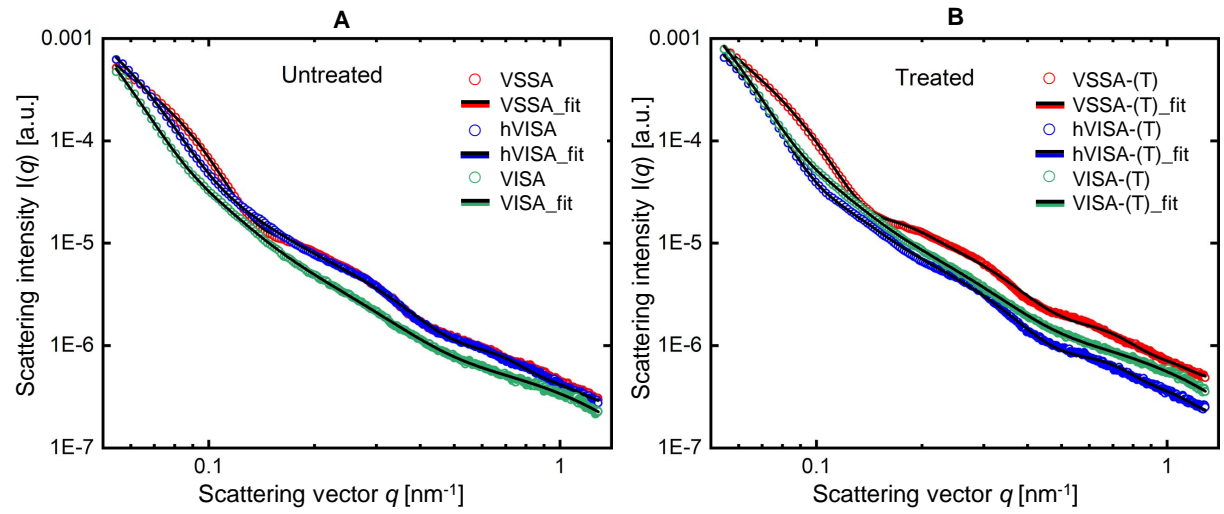

Fig. 1 Comparison of small angle X-ray scattering (SAXS) curves of vancomycin untreated cells (A) and treated cells (B) of VSSA, hVISA, and VISA groups.

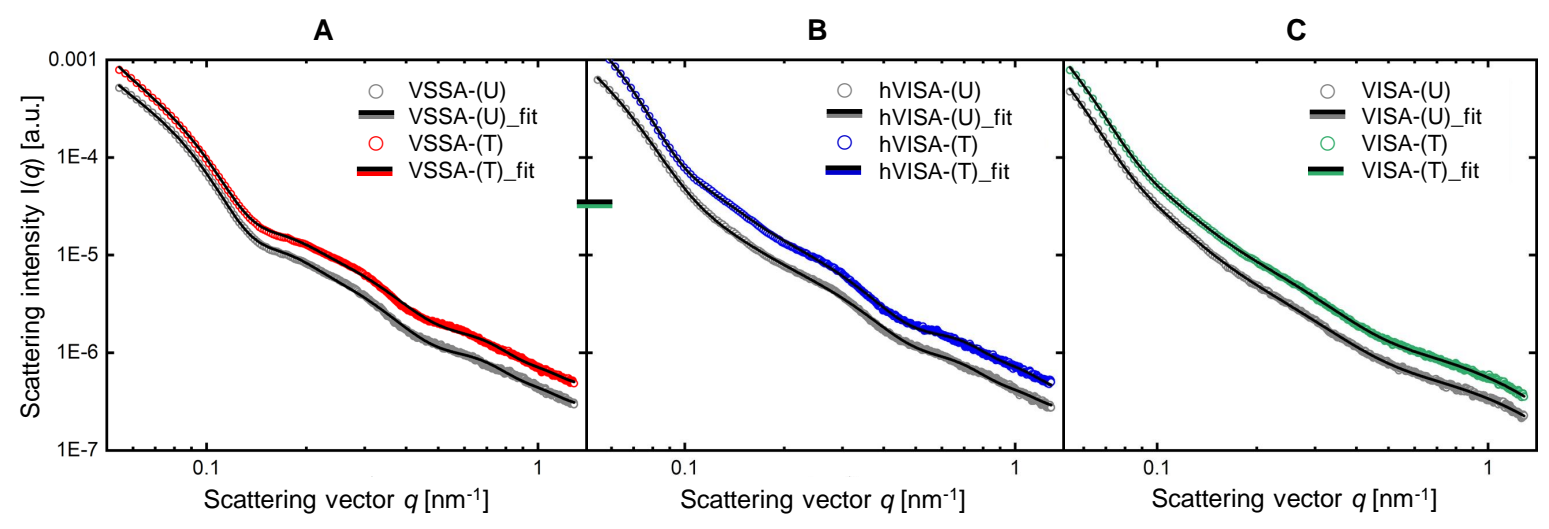

Fig. 2 Comparison of small angle X-ray scattering (SAXS) between vancomycin untreated cells and treated cells of VSSA (A), hVISA (B), and VISA (C) groups.

and PAP-AUC value may have indirect effect to their different SAXS performance. However, further study is required for verification.

For the intracellular components generated from the SAXS scattering curves, significant differences were found among the bacterial groups. Under the untreated condition, the ribosome of VSSA was significantly smaller than that of the hVISA suggesting that they might have discrepancy in performances even under condition without vancomycin pressure, as they

Table 2 Size distribution of internal composition of $S$. aureus modeled from SAXS scattering.

\begin{tabular}{llccccc}
\hline \multirow{2}{*}{ Phenotype } & \multicolumn{7}{c}{ Size distribution"(nm) \pm SD } \\
\cline { 2 - 7 } & 1 & $p$-value & 2 & $p$-value & 3 & $p$-value \\
\cline { 2 - 7 } VSSA-(U) & $58.8 \pm 5.2$ & 0.279 & $17.1 \pm 0.1$ & 0.060 & $3.4 \pm 0.6$ & 0.144 \\
VSSA-(T) & $65.5 \pm 7.6$ & & $14.3 \pm 0.1$ & & $4.3 \pm 0.5$ & 0.443 \\
\hline hVISA-(U) & $72.1 \pm 10.9$ & 0.389 & $17.1 \pm 0.2$ & $1.8 \pm 0.5$ & \\
hVISA-(T) & $80.3 \pm 9.9$ & & $17.1 \pm 0.2$ & & $2.2 \pm 0.5$ & 0.818 \\
\hline VISA-(U) & $92.2 \pm 13.8$ & 0.748 & $11.0 \pm 1.7$ & 0.408 & $1.6 \pm 0.3$ & \\
VISA-(T) & $88.7 \pm 12.7$ & & $12.3 \pm 1.7$ & & $1.5 \pm 0.3$ & \\
\hline
\end{tabular}

" Size distributions were calculated by the average diameter in nanometer: 1 = sphere model for ribosome; $2=$ sphere model for DNA; 3 = sphere model for small proteins (a few nanometers); VSSA, vancomycin-susceptible $S$. aureus; hVISA, heterogeneous vancomycin-intermediate $S$. aureus; VISA, vancomycin-intermediate $S$. aureus; T, treated with vancomycin; $\mathrm{U}$, untreated with vancomycin. 
Table 3 Comparing the size of sphere models between the bacterial groups under vancomycin untreated and treated conditions.

\begin{tabular}{llccccc}
\hline Comparison & Size & \multicolumn{2}{c}{ Size distribution $(\mathrm{nm}) \pm$ SD } & & \multicolumn{2}{c}{$p$-value } \\
\cline { 3 - 4 } \cline { 5 - 6 } & & Untreated & Treated & & Untreated & Treated \\
\hline \multirow{2}{*}{ VSSA-hVISA } & 1 & $58.8 \pm 5.2$ vs. $72.1 \pm 10.9$ & $65.5 \pm 7.6$ vs. $80.3 \pm 9.9$ & & 0.00001 & 0.108 \\
& 2 & $17.1 \pm 0.1$ vs. $17.1 \pm 0.2$ & $14.3 \pm 0.1$ vs. $17.1 \pm 0.2$ & & 0.405 & 0.088 \\
& 3 & $3.4 \pm 0.6$ vs. $1.8 \pm 0.5$ & $4.3 \pm 0.5$ vs. $2.2 \pm 0.5$ & 0.029 & 0.070 \\
\hline \multirow{3}{*}{ VSSA-VISA } & 1 & $58.8 \pm 5.2$ vs. $92.2 \pm 13.8$ & $65.5 \pm 7.6$ vs. $88.7 \pm 12.7$ & 0.017 & 0.060 \\
& 2 & $17.1 \pm 0.1$ vs. $11.0 \pm 1.7$ & $14.3 \pm 0.1$ vs. $12.3 \pm 1.7$ & & 0.004 & 0.109 \\
& 3 & $3.4 \pm 0.6$ vs. $1.6 \pm 0.3$ & $4.3 \pm 0.5$ vs. $1.5 \pm 0.3$ & 0.110 & 0.001 \\
\hline \multirow{2}{*}{ hVISA-VISA } & 1 & $72.1 \pm 10.9$ vs. $92.2 \pm 13.8$ & $80.3 \pm 9.9$ vs. $88.7 \pm 12.7$ & & 0.119 & 0.446 \\
& 2 & $17.1 \pm 0.2$ vs. $11.0 \pm 1.7$ & $17.1 \pm 0.2$ vs. $12.3 \pm 1.7$ & & 0.004 & 0.080 \\
& 3 & $1.8 \pm 0.5$ vs. $1.6 \pm 0.3$ & $2.2 \pm 0.5$ vs. $1.5 \pm 0.3$ & & 0.140 & 0.115 \\
\hline
\end{tabular}

"Size distributions were calculated by the average diameter in nanometer: 1 = sphere model for Ribosome; $2=$ sphere model for DNA; 3 = sphere model for small proteins (a few nanometers); VSSA, vancomycin-susceptible $S$. aureus; hVISA, heterogeneous vancomycin-intermediate $S$. aureus; VISA, vancomycin-intermediate $S$. aureus; Treated, treated with vancomycin; Untreated, untreated with vancomycin.

had different PAP-AUC values. However, no significant difference under vancomycin treated condition was found. This finding suggested a different response of the bacteria to vancomycin. It is possible that the vancomycin susceptible cells (VSSA) has higher stress response to vancomycin than those of the vancomycin non-susceptible (hVISA and VISA) cells leading to a drastic change of ribosome in the VSSA cells to be similar to those of the vancomycin non-susceptible (hVISA and VISA). (The ribosomes of the hVISA and the VISA were rarely changed.) As a result, no difference of ribosome among these three bacterial groups under the treated condition (Table 3 ). In addition, the ribosome and DNA of VSSA vs. VISA, and the DNA of hVISA vs. VISA were different only under the untreated condition, suggesting that even in the condition with no pressure from vancomycin, the VISA cells have usual different performance from those of the VSSA and the hVISA.

Interestingly, the sizes of ribosomes and small proteins in the VSSA and the hVISA groups increased after exposed to vancomycin, in contrast to the reducing size in the VISA group. This may be due to the VSSA and the hVISA cells had higher stress response to vancomycin for their survival, whereas the VISA cells could continue their life cycles as usual. However, further investigation with larger sample size is needed to verify and to understand more on the bacterial performance.

Though the target site of vancomycin is D-alanylD-alanine residue of the cell wall [23], it may have indirect effect to other intracellular components of the bacterial cells such as cell membrane, ribosome, and DNA [23]. The VISA and the hVISA cells had thickening cell wall and increasing free D-alanyl-Dalanine residues, leading to vancomycin blockage from reaching its target site $[21,24]$. This may be the reason of lower stress response of the VISA and the hVISA to vancomycin than that of the VSSA. The SAXS is a useful technique for investigating the changes of intracellular components of bacteria under the effects of antimicrobials. The different degree in stress responses may be strategically applied to combat the low-level vancomycin resistant bacteria like hVISA and VISA.

Since this is the preliminary study with a limitation of sample size, further studies with larger sample size of each bacterial group, testing under various vancomycin concentrations, and using various antimicrobials are necessary. The studies' results could be used to elucidate the adaptation of bacterial intracellular components during the antimicrobial stress response.

\section{CONCLUSION}

This is a preliminary study of VSSA, hVISA, and VISA cellular changes under stress from vancomycin compared with the non-stress treated cells. Using the SAXS, scattering patterns were different among the vancomycin susceptible and non-susceptible $S$. aureus. The SAXS can be used as a method to study the effects of antimicrobials on bacteria to investigate the changes of bacterial ultra-structures.

Acknowledgements: This project was supported by Khon Kaen University Research Grant of Synchrotron Light Research Promotion for new user, KKU (project No. KKUSyn60006). Part of this project was support by a research grant from Khon Kaen University (Project No. 620017).

\section{REFERENCES}

1. Kinney KK (2010) Treatment of infections caused by antimicrobial-resistant gram-positive bacteria. Am J Med Sci 340, 209-217.

2. Fong RK, Low J, Koh TH, Kurup A (2009) Clinical features and treatment outcomes of vancomycinintermediate Staphylococcus aureus (VISA) and het- 
eroresistant vancomycin-intermediate Staphylococcus aureus (hVISA) in a tertiary care institution in Singapore. Eur J Clin Microbiol Infect Dis 28, 983-987.

3. Khatib R, Jose J, Musta A, Sharma M, Fakih MG, Johnson LB, Riederer K, Shemes S (2011) Relevance of vancomycin-intermediate susceptibility and heteroresistance in methicillin-resistant Staphylococcus aureus bacteraemia. J Antimicrob Chemother 66, 1594-1599.

4. Howden BP, Johnson PD, Ward PB, Stinear TP, Davies JK (2006) Isolates with low-level vancomycin resistance associated with persistent methicillin-resistant Staphylococcus aureus bacteremia. Antimicrob Agents Chemother 50, 3039-3047.

5. Rose WE, Fallon M, Moran JJM, Vanderloo JP (2012) Vancomycin tolerance in methicillin-resistant Staphylococcus aureus: influence of vancomycin, daptomycin, and telavancin on differential resistance gene expression. Antimicrob Agents Chemother 56, 4422-4427.

6. Howden BP, Davies JK, Johnson PD, Stinear TP, Grayson ML (2010) Reduced vancomycin susceptibility in Staphylococcus aureus, including vancomycinintermediate and heterogeneous vancomycinintermediate strains: resistance mechanisms, laboratory detection, and clinical implications. Clin Microbiol Rev 23, 99-139.

7. Graewert MA, Svergun DI (2013) Impact and progress in small and wide angle X-ray scattering (SAXS and WAXS). Curr Opin Struct Biol 23, 748-754.

8. Blanchet CE, Svergun DI (2013) Small-angle X-ray scattering on biological macromolecules and nanocomposites in solution. Annu Rev Phys Chem 64, 37-54.

9. Filippov SK, Franklin JM, Konarev PV, Chytil P, Etrych T, Bogomolova A, Dyakonova M, Papadakis CM, et al (2013) Hydrolytically degradable polymer micelles for drug delivery: A SAXS/SANS kinetic study. Biomacromolecules 1411, 4061-4070.

10. Von Gundlach AR, Garamus VM, Willey TM, Ilavsky J, Hilpert K, Rosenhahn A (2016) Use of small-angle X-ray scattering to resolve intracellular structure changes of Escherichia coli cells induced by antibiotic treatment. $J$ Appl Crystallogr 49, 2210-2216.

11. CLSI (2019) Methods for Dilution Antimicrobial Susceptibility Tests for Bacteria That Grow Aerobically. CLSI document M07-A9, 16th edn, Clinical and Laboratory Standards Institute, Wayne, PA.

12. Wootton M, Howe RA, Hillman R, Walsh TR, Bennett PM, MacGowan AP (2001) A modified population analysis profile (PAP) method to detect hetero-resistance to vancomycin in Staphylococcus aureus in a UK hospital. $J$ Antimicrob Chemother 47, 399-403.
13. Soontaranon S, Rugmai S (2012) Small Angle X-ray Scattering at Siam Photon Laboratory. Chin J Phys 50, 204-210.

14. Breßler I, Kohlbrecher J, Thünemann AF (2015) SASfit: a tool for small-angle scattering data analysis using a library of analytical expressions. J Appl Crystallogr 48, 1587-1598.

15. Craievich AF (2002) Synchrotron SAXS studies of nanostructure materials and colloidal solutions. A Review. Mat Res 5, 1-11.

16. Tosi T, Hoshiga F, Millership C, Singh R, Eldrid C, Patin D, Mengin-Lecreulx D, Thalassinos K, et al (2019) Inhibition of the Staphylococcus aureus c-di-AMP cyclase DacA by direct interaction with the phosphoglucosamine mutase GlmM. PLoS Pathog 15, e1007537.

17. Capp JA, Hagarman A, Richardson DC, Oas TG (2014) The statistical conformation of a highly flexible protein: small-angle X-ray scattering of $S$. aureus protein A. Structure 22, 1184-1195.

18. von Gundlach AR, Ashby M, Gani J, Lopez-Perez PM, Cookson A, Huws S, Rumancev C, Garamus VM, et al (2019) BioSAXS measurements reveal that two antimicrobial peptides induce similar molecular changes in Gram-negative and Gram-positive bacteria. Front Pharmacol 10, ID 1127.

19. Hiramatsu K, Kayayama Y, Matsuo M, Aiba Y, Saito M, Hishinuma T, Iwamoto A (2014) Vancomycinintermediate resistance in Staphylococcus aureus. J Glob Antimicrob Resist 2, 213-224.

20. Sirichoat A, Wongthong S, Kanyota R, Tavichakorntrakool R, Chanawong A, Welbat JU, Lulitanond A (2016) Phenotypic characteristics of vancomycin-nonsusceptible Staphylococcus aureus. Jundishapur J Microbiol 9, e26069.

21. Cui J, Zhang H, Mo Z, Yu M, Liang Z (2021) Cell wall thickness and the molecular mechanism of heterogeneous vancomycin-intermediate Staphylococcus aureus. Lett Appl Microbiol, 72, 604-609.

22. Cui L, Ma X, Sato K, Okuma K, Tenover FC, Mamizuka EM, Gemmell CG, Kim MN, et al (2003) Cell wall thickening is a common feature of vancomycin resistance in Staphylococcus aureus. J Clin Microbiol 41, 5-14.

23. Reynolds PE, Somner EA (1990) Comparison of the target sites and mechanisms of action of glycopeptide and lipoglycodepsipeptide antibiotics. Drugs Exp Clin Res 16, 385-389.

24. Cui L, Iwamoto A, Lian JQ, Neoh HM, Maruyama T, Horikawa Y, Hiramatsu K (2006) Novel mechanism of antibiotic resistance originating in vancomycinintermediate Staphylococcus aureus. Antimicrob Agents Chemother 50, 428-438. 